\title{
Teaching Delta-Sigma Signal Conversion in an Interfacing Course
}

\author{
Witold Kinsner \\ Department of Electrical and Computer Engineering \\ University of Manitoba, Winnipeg, MB, Canada R3T 5V6 \\ $<$ witold.kinsner@umanitoba.ca>
}

\begin{abstract}
Conversion of signals is fundamental to the interfacing of embedded systems. Such signal conversions include (i) analog-to digital $(A / D)$ in order to translate an analog form of the signal to its sampled and quantized form for digital signal processing, (ii) digital-to-analog (D/A) in order to translate the digital samples to a corresponding boxcar signal for further low-pass filtering and recovery of the original signal, and (iii) digital-to-digital (D/D) to achieve new desired properties of the data.

This paper focuses on teaching the delta-sigma $(\Delta \Sigma)$ $A / D$ conversion that is often omitted from an interfacing course because it appears to be a difficult topic to comprehend and to teach. This new approach links the new $\Delta \Sigma$ conversion to the other classes of $A / D$ conversion techniques explicitly, thus unifying and simplifying the teaching of signal conversions.
\end{abstract}

Keywords: Interfacing in embedded systems; signal converters; teaching delta-sigma conversions.

\section{INTRODUCTION}

Conversion of signals is fundamental to the interfacing of embedded systems [3], and particularly microcontrollers ( $\mu \mathrm{c})$ [9], [26], [33], microprocessors ( $\mu \mathrm{P})$ [32], and microcomputers $(\mu \mathrm{C})$ [18]. The signal conversions include (i) analog-to digital (A/D) in order to translate an analog form of the signal to its sampled and quantized form for digital signal processing, (ii) digital-to-analog (D/A) in order to translate the digital samples to a corresponding boxcar signal for further low-pass filtering and recovery of the original signal, and (iii) digital-to-digital (D/D) to achieve new desired properties of the data such as (a) elimination of a DC component, (b) self-clocking by embedding the transmission clock into the transmitted data, and (c) polarity independence to eliminate the need to colour the wires in a data-carrying cable.

A course on microcontroller interfacing must include all those conversions, in addition to many other pertinent topics [16], [17], [32], [4]. This paper describes such a course, with a focus on teaching the delta-sigma $(\Delta \Sigma)$ conversion that is often omitted because it appears to be the most difficult topic to comprehend and to teach (e.g., [12], [29], [2]).

CEEA Conf. 2014; Paper 107

Canmore, AB; June 8-11, 2014
The most important contribution of this paper is the approach to teaching the $\Delta \Sigma$ conversion in the context of the evolution of ideas related to the operation of other more common techniques such as parallel (flash and its variations) and serial, either linear on nonlinear. The serial signal $\mathrm{A} / \mathrm{D}$ and $\mathrm{D} / \mathrm{A}$ converters (ADC and DAC) are often covered in a course on interfacing, and include the following types (i) counting (single ramp), (ii) tracking, (iii) successive approximation (SA), (iv) integrating (dual slope, quad slope), and (v) voltage-to-frequency (V/F)-based converters [16]. The first four types can be classified as voltage-to-timegate (V/T)-based converters. The $\mathrm{V} / \mathrm{F}$ converter can be treated as a logical dual of the $\mathrm{V} / \mathrm{T}$ converter, but with several new very desirable features [16].

Once these relations are established, we can combine the best features of the $\mathrm{V} / \mathrm{F}$ and $\mathrm{V} / \mathrm{T}$ converters, and add concepts from signal processing and control to achieve noise shaping through dithering (spectrum spreading). Such $\Delta \Sigma$ converters can attain unprecedented resolution. This paper presents the roots of the $\Delta \Sigma$ conversion as found in the differential pulse-coded modulation (PCM), as well as its theory, implementation, and benefits to embedded systems.

\section{STANDARD ADC CONCEPTS}

\subsection{Data Acquisition System}

2.1.1 Types of Signals: An analog-to-digital (A/D) converter (ADC) is an electronic circuit that converts an analog signal (continuous in both time and magnitude) to a discrete signal (discrete in time, but continuous in magnitude) and to a digital signal (discrete in both time and magnitude), as shown in Fig. 1.

While the discrete signal is the same as the analog signal at time $j$, the digital signal approximates the discrete signal to one least-significant bit (LSB), equal to the quantization step, $Q$. The $\mathrm{Q}$ step is the interval of uncertainty in the ADC, and is equivalent to the leastsignificant bit (LSB), and is given by

$$
Q=\frac{\mathrm{FS}}{2^{N}} \equiv 1 \mathrm{LSB}
$$


where FS is the full scale (the peak-to-peak) value of the signal), $v(t)=V_{\text {max-pp}}$, and $N$ is the number of bits in a sample.

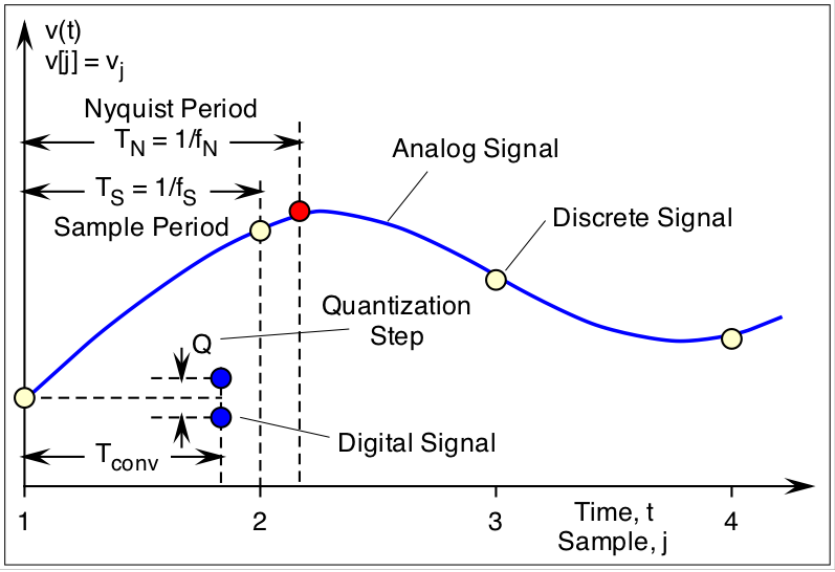

Fig 1. Impact of oversampling by a factor $M$.

2.1.2 Signal Acquisition and Conversion: The standard way to obtain the discrete signal is to use a sample-andhold $(\mathrm{S} \& \mathrm{H})$ circuit that remembers the analog discrete signal value $v_{j}, \equiv v[j]=v(t)$ as captured at time $j=t_{j}=t$, where the symbol " $\equiv$ " reads "is equivalent to." The S\&H must be selected to hold the value between successive samples with a negligible droop, i.e., $v_{\text {droop }}[j] \leq$ Q. If this condition is satisfied, then the conversion time $T_{\text {conv }}$ can extend up to the sampling time, $T_{\mathrm{S}}$,

$$
T_{\text {conv }} \leq T_{S}<T_{N}
$$

When the S\&H device is not used (due to its expense) and the analog input signal is fed directly to the ADC, another constraint must be considered: the signal must change slowly so that it does not exceed one quantization step during the conversion time. This critical time is called the aperture time, $t_{\mathrm{a}}$. It can be shown that the aperture time can be calculated from [Kins13a]

$$
\left(t_{a}\right)_{\min }=\frac{F S}{2^{N}\left(\frac{d v(t)}{d t}\right)_{\max }}
$$

where $(\bullet)_{\max }$ denotes the maximum time derivative of the analog signal. Thus, the conversion time must be faster than the minimum aperture time

$$
T_{\text {conv }} \leq t_{a \min }<T_{N}
$$

2.1.3 The ADC: As shown in Fig. 2, the ADC takes an analog signal, converts it to the digital form, and transfers the data to a digital signal processor. The ADC can have any of the forms listed in the previous section, such as the SA type that is not as fast as the flash converter, but can also convert in a fixed amount of time, proportional to the number of bits, $N$, in a sample. The ADC requires a sampling clock and a voltage reference.

2.1.4 The Analog LPF: As shown in Fig 2, the ADC is preceded by an analog low pass filter (LPF), also called the anti-aliasing filter (AAF). The filter is necessary to establish a cutoff frequency, $f_{c}$, defined at the 3-dB power spectrum density $\mathrm{P}(\mathrm{f})$ dropoff, as well as the rolloff frequency, $f_{R}$, $\equiv f_{B}$ that defines the bandwidth, $B$, of the baseband signal. The rolloff frequency is defined at an appropriate level of the floor noise.

The cutoff frequency, $f_{c}$, is used to calculate the Nyquist (minimum) sampling frequency, $f_{N}$, required to reconstruct the signal without losses

$$
f_{N}=2 f_{c}
$$

The actual sampling frequency is selected from the worstcase rolloff frequency

$$
f_{S}=2 f_{B}>f_{N}
$$

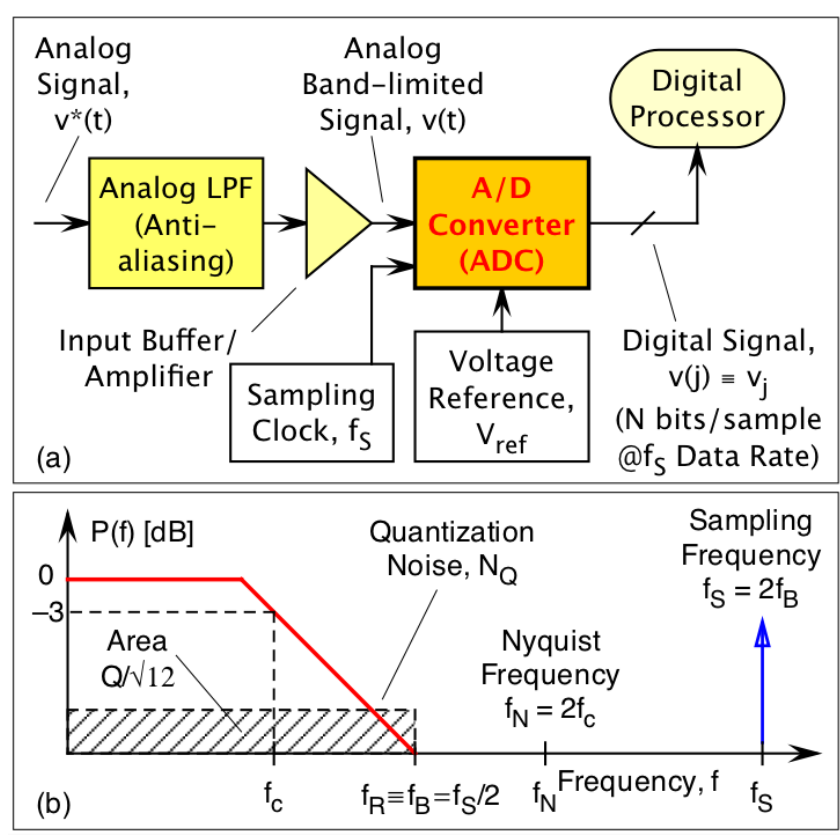

Fig 2. (a) A band-limited signal is converted to digital samples, each with $\mathrm{N}$ bits, at a rate of the sampling frequency, $f_{S}$. (b) Definitions of sampling frequency. 
Notice that the sampling should never be done at the Nyquist frequency in order to avoid phantom DC reconstruction (when the samples are all zero at the zero crossings of the periodic signal). As shown in Fig. $1, f_{S}>f_{N}$, or $T_{S}<T_{N}$. For example, the telephone-quality speech has a bandwidth from $300 \mathrm{~Hz}$ to $3,300 \mathrm{~Hz}$. Thus, the Nyquist frequency is $f_{N}=2 \times 3300=6600 \mathrm{sps}$ (samples per second). The sampling is done at $f_{S}=8 \mathrm{ksps}$ (kilosamples per second), which is over $20 \%$ higher than $f_{N}$.

2.1.5 Implementation Issues: Designing the LPF is not trivial, as steep-skirt filters are difficult to implement, and may become oscillators (e.g., [21, Fig. 6]).

In high-speed systems, a buffer is also necessary between the LPF and the ADC to separate the filter from the converter in terms of driving capabilities and frequency independence (e.g., [22], [20]).

The sampling clock feeding the ADC must also be designed for stability and low jitter to reduce noise (e.g., [21]). Still another part of the circuit is a high-accuracy full-scale voltage reference required by the ADC. It can be designed using a pulse-width modulation (e.g., [23], [24]).

\section{Quest To REDUCE $N_{Q}$}

The impact of the quantization noise, $N_{Q}$, on the number of bits is very pronounced [34]. Spreading of the noise over a wider bandwidth has worked in many signal processing areas, and has been applied in the ADC area.

\subsection{Theoretical SNR}

The signal to noise ratio, SNR, is defined as the ratio of the useful signal to the unwanted noise in the system. The ratio can be expressed in terms of power or magnitude. This measure is very important as it is linked to many performance characteristics of a communications or signal processing system. One of the noise components in a data acquisition system is its unavoidable quantization noise, $N_{Q}$

The ADC can be linear or nonlinear [Kins13a], as defined by its signal transfer characteristic (STC). The $\mathrm{ADC}$ is said to be linear if all the quantization steps, $Q$, are equal. The maximum quantization error (noise) is $N_{Q \max }=$ $\mathrm{Q}$ or $N_{Q \max }= \pm \mathrm{Q} / 2$, depending on the ADC implementation. For that maximum quantization error, root-mean squared value of the error is [Kins13a]

$$
N_{Q r m s}=\frac{Q}{\sqrt{12}}=\frac{Q}{2 \sqrt{3}} \approx \frac{Q}{3}
$$

Since all the frequencies in the bandwidth $B$ are equally probable, the distribution of the quantization noise is uniform. For a full-scale sine-wave, the theoretical SNR is [15]

CEEA Conf. 2014; Paper 107

Canmore, AB; June 8-11, 2014

$$
\mathrm{SNR}=6.02 N+1.76[\mathrm{~dB}]
$$

\subsection{Oversampling to Reduce $N_{Q}$}

Since the quantization noise, $N_{Q}$, affects the performance of a system, can its impact be reduced? Yes, the impact can be reduced if the signal is oversampled [5] by a factor $M=f_{S} /\left(2 f_{B}\right)$, as shown in Fig. 3 .

The quantization noise is reduced because it is now spread over a wider bandwidth, and much of the noise is removed by a LPF which can now be a digital filter. Most of such digital filters are the finite-impulse response (FIR) LPF because their phase response is linear (e.g., Laco07, Fig. 7]). Digital filters with very steep skirts can be implemented reliably (e.g., [25], [30]).

The digital LPF is followed by a decimation stage to reduce the rate of data from the oversampled $M f_{S}$ to the original sampling rate, $f_{S}$.

An added advantage of oversampling is the much flatter rolloff of the analog LPF that can now be implemented with a lower-order LPF.

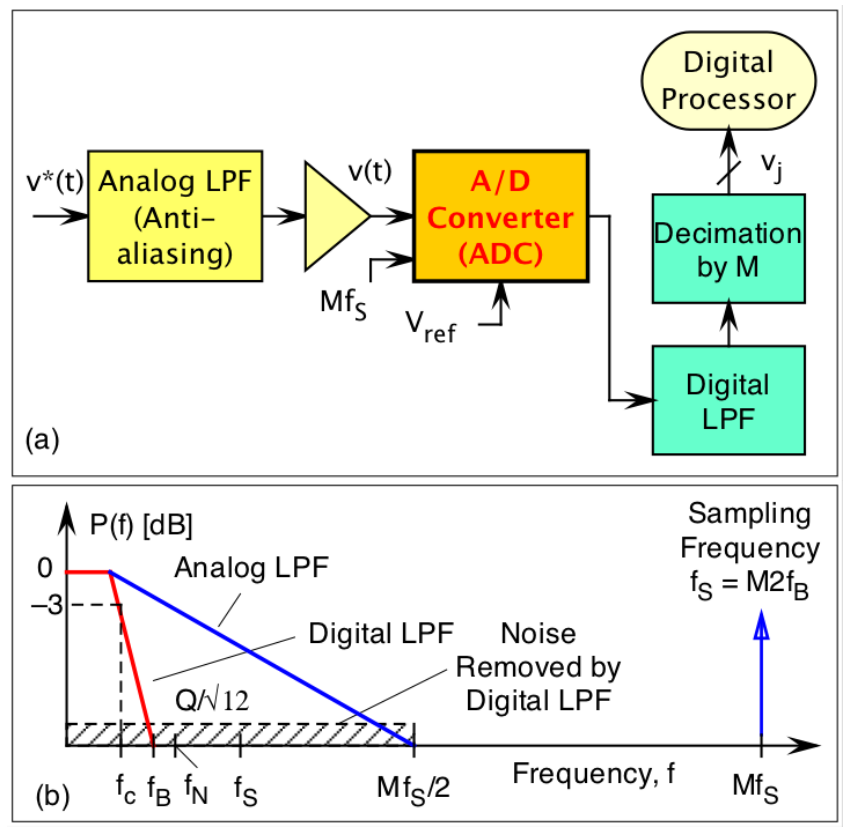

Fig 3. Impact of oversampling by a factor $M$.

This oversampling improves the SNR by $10 \log _{10}(M)$. However, this improvement is not very efficient because the spreading is still uniform. To improve the SNR by 6.02 $\mathrm{dB}(n=1 \mathrm{bit})$, we must oversample by $M=4$. The second bit improvement $(n=2)$ requires $M=16$, and the third bit $(n=3), M=64$, or in general, $M=2^{m}$ where $m=2^{n}$. 


\subsection{Noise Shaping to Reduce $N_{Q}$}

In order to improve the SNR more efficiently, we should abandon the uniform spreading of the $N_{Q}$ distribution in favour of a non-uniform $N_{Q}$ distribution. This process is called noise shaping. If we could shape the distribution so that it could be skewed to the right, then the LPF would remove a much larger portion of the unwanted part of the noise.

Figure 4 shows the noise shaping for different degrees of complexity. The uniform spectrum of the noise transfer function (NTF) represents the spreading due to the standard oversampling on any ADC [19]. The nonlinear first-order shaping skews the spectrum to the right, so less noise is retained in the bandwidth $\mathrm{B}$ of interest (where the curves intersect). Higher-order shaping provides even more gain.

In order to achieve this non-linear noise shaping, oversampling alone will not be sufficient. We must introduce some sort of feedback so that past values of the noise could be reused not just once, but many times in an iterative cascade cycle, as discussed next.

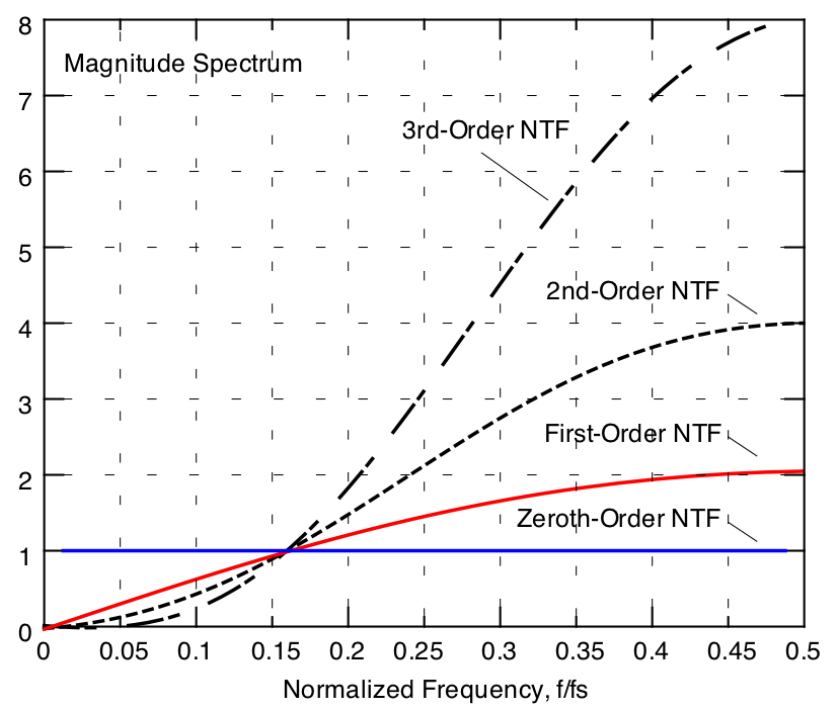

Fig 4. Quantization noise shaping. (After [1, Fig. 12])

\section{4. $\Delta \Sigma$ CONCEPTS}

\subsection{Evolution of Basic Concepts}

The delta-sigma $(\Delta \Sigma)$ analog-to-digital (ADC) is used in many applications such as voice, audio, and highresolution measurements. Such devices are slower than flash and successive approximation (SA), but have a much higher effective resolution.

The $\Delta \Sigma$ concept was developed in France in 1946 [7], US Bell Labs in 1952 [6], and Holland's Phillips Labs in 1952 [11]. The key ideas and historical evolutions is discussed by Hauser [10], Azis et al. [1], Kester [12], [13], [14], and Wooley [35].

The objective of the $\Delta \Sigma$ ADC is the same as before: convert an analog input signal in the range $\left[v_{\min }, v_{\max }\right]$ to its digital equivalent with a resolution of $\mathrm{N}$ bits per sample, while satisfying the Nyquist-sampling and aperture-time constraints.

The key concepts behind the $\Delta \Sigma$ ADC are: (i) oversampling, (ii) quantization noise spreading and shaping, (iii) digital filters, and (iv) decimation.

\subsection{Classes of Converters}

4.2.1 PWM-Modulator-Based ADC: The majority of standard serial $\mathrm{A} / \mathrm{D}$ converters change the sampled signal into a time gate whose duration is proportional to the magnitude of the sampled signal. The single time-gategeneration part of the ADC can be considered a pulsewidth modulator (PWM). During the time-gate pulse, clock pulses are counted. Since the clock frequency, $T_{C K}$, is constant, the clock count is proportional to the sampled signal, $v_{j}$. The clock frequency is selected to reach the maximum count for the maximum duration of the time gate. This scheme is illustrated in Fig. 5a.

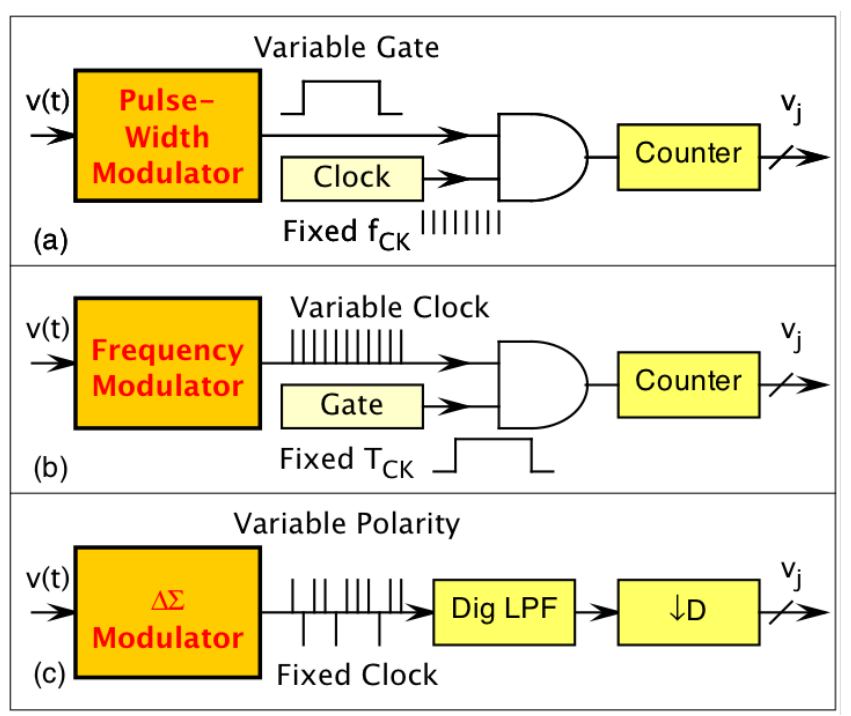

Fig 5. Classes of serial ADC schemes. (a) PWM-based ADC. (b) FM-based ADC. (c) $\Delta \Sigma$ M-based ADC.

4.2.2 Frequency-Modulator-Based ADC: Figure 5b shows the second class of ADC devices. They convert the sampled signal into a unipolar pulse stream whose frequency is proportional to the magnitude of the sampled signal. This charge-pump device can be considered as frequency modulator (FM), and is called voltage-to-frequency $(\mathrm{V} / \mathrm{F})$ converter. This pulse stream has a low rate for low voltage, and a high rate for a high voltage. The pulse stream can be considered to be a variable clock which is counted during a 
constant time gate. The duration of the gate is calculated so that the counter reaches its maximum for the highest frequency of the pulses.

4.2.3 Delta-Sigma-Based ADC: The $\Delta \Sigma$ scheme is an extension of this $\mathrm{V} / \mathrm{F}$ converter in which the sampled input signal (scaled to $[-1,1]$ for convenience) is also converted to a pulse stream, but now bipolar, with a fixed frequency, a fixed amplitude of -1 or +1 , and a fixed duration of $\tau$. This part of the converter is called the delta-sigma $(\Delta \Sigma)$ modulator (DSM). The pulse stream is filtered out by a digital LPF, and its output is decimated to obtain the desired data, as shown in Fig. 5c.

\subsection{Selection of Pulse Polarity}

The polarity of the pulses is selected in such a way that the time average of the pulse stream converges to the sampled input signal to an arbitrary degree of accuracy. The more pulses generated in the each interval $T_{S}$, the greater the accuracy and precision. This is illustrated in Fig. 6.

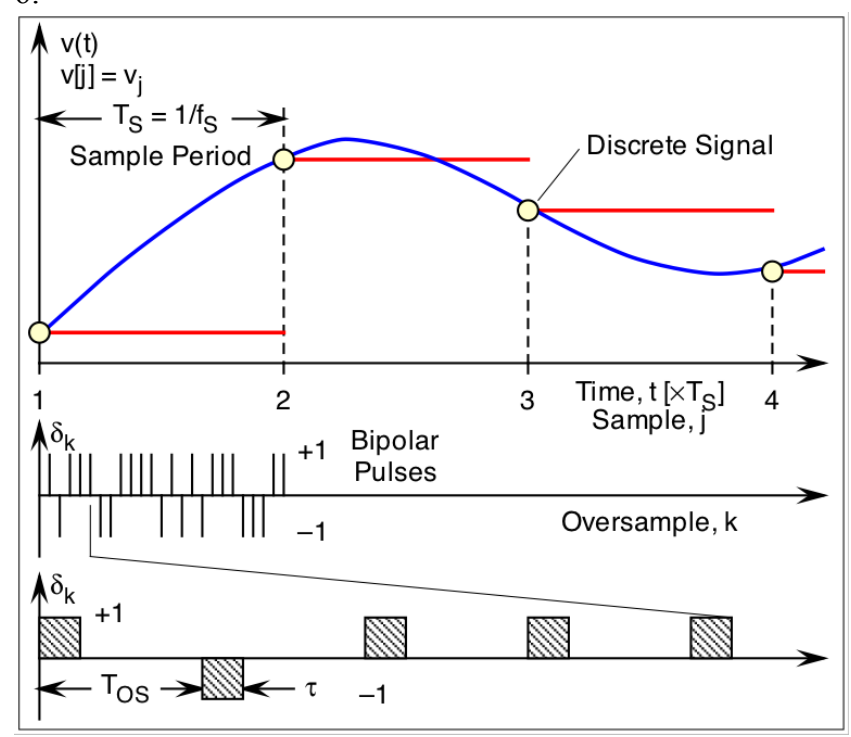

Fig 6. A unity bipolar pulse stream representing a sample $v_{j}$.

To demonstrate the simplicity of the idea, let us consider the desired equality between the input signal sample $v_{j}$ and a measure $\mu_{j}$ of the bipolar pulse stream between two successive discrete samples

$$
v_{j}=\left\langle\delta_{k}\right\rangle_{j} \triangleq \mu_{j}\left(\delta_{k}\right)
$$

A simple measure is the average of the $M$ pulses, each of duration $\tau$ within each sample interval $T_{S}$. For example (Fig. 6 ), the oversampling is $M=24$ (i.e., 15 positive pulses and 9 negative pulses). Since the arithmetic sum of the pulses is 6 , the measure is $\mu_{1}=6 / 24=1 / 4$. In general, this can be written as

$$
\mu_{j}\left(\delta_{k}\right)=\frac{1}{M \tau} \sum_{k=1}^{M} \delta_{k} \tau=\frac{1}{M} \sum_{k=1}^{M} \delta_{k} \equiv \frac{S_{j M}}{M}
$$

where $S_{j M}$ denotes the total sum of the pulses in the $j$ th sample interval. It is seen from (9) that the duration of the pulses does not matter (i.e., the pulses can be as narrow as practicable, or can reach the entire pulse period $T_{O S}$.

\subsection{Sequence Generation}

The key question is how the pulse sequence can be generated. Observe that at the end of the sampling period $T_{S}$, all $M$ pulses are counted, and the following equality should hold

$$
v_{j}=\frac{S_{j M}}{M} \text { or } v_{j}-\frac{S_{j M}}{M}=0
$$

or

$$
M v_{j}-S_{j M}=0
$$

This expression provides a clue about the pulse steam formation during the sampling interval for $k<M$. That is, after counting $k$ pulses, if the partial sum, $S_{j k}$, is too small with respect to $k v_{j}$, the next pulse should be +1 . That is,

$$
\Delta_{j k}=k v_{j}-S_{j k}>0 \text { then } \delta_{k+1}:=1
$$

On the other hand, if the partial sum is too large (or equal), the next pulse should be

$$
\Delta_{j k}=k v_{j}-S_{j k} \leq 0 \text { then } \delta_{k+1}:=-1
$$

This strategy should produce a pulse stream within each sample interval $T_{S}$ that converges to the value of the corresponding input signal sample $v_{j}$, provided $\mathrm{M}$ is sufficiently large. This can be written as a C-like pseudocode, and shown in Algorithm 1 (e.g., [27]).

Algorithm 1: Pseudocode of the $\Delta \Sigma$ modulator.

\begin{tabular}{|l|l|}
\hline Step & Code \\
\hline 1 & $\mathrm{k}=1 ;$ \\
\hline 2 & while $(1)\{$ \\
\hline 3 & $\mathrm{~S} \mathrm{k}=$ compute_partial_S_k ()$;$ \\
\hline 4 & if $\left(\mathrm{k}{ }^{*} \mathrm{v} \_\mathrm{j}-\mathrm{S} \mathrm{j} \_\mathrm{k}>0\right.$ \\
\hline 5 & output $(1) ;$ \\
\hline 6 & else \\
\hline 7 & output $(-1) ;$ \\
\hline 8 & $\mathrm{k}++;$ \\
\hline 9 & 3 \\
\hline
\end{tabular}




\subsection{Block Diagram of $\Delta \Sigma$ Modulator}

Algorithm 1 can be translated into a block diagram shown in Fig. 7. It is clear why the modulator is called $\Delta \Sigma$. Some literature considers this structure as $\Sigma \Delta$ (summer followed by the difference maker).

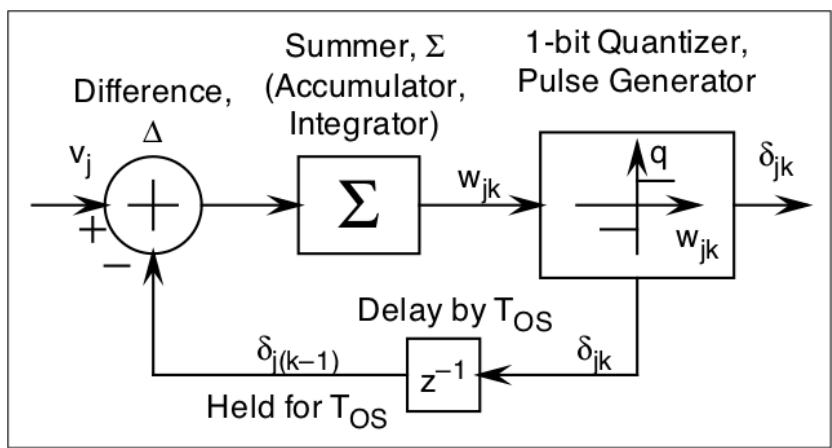

Fig 7. Fundamental block diagram of the $\Delta \Sigma$ modulator.

Notice that the above diagram appears to be different from most $\Delta \Sigma$ modulators reported in literature. The alternative diagrams substitute the summer block $(\Sigma)$ with an analog integrator $\left(\int\right)$, the 1-bit quantizer with a 1-bit $\mathrm{A} / \mathrm{D}$ converter, and the sample delay $\left(z^{-1}\right)$ with a 1-bit D/A converter. The alternative diagrams represent a more generalized $\Sigma \Delta$ modulator. Our diagram may be easier to understand, at least initially during teaching in a course for a diverse audience.

\subsection{Verification of the $\Delta \Sigma$ Modulator}

Table 1 lists several steps that follow the $\Delta \Sigma$ algorithm and the bock diagram. It is intended to re-inforce the concept in a class.

Table 1: $\Delta \Sigma$ sequence generation.

\begin{tabular}{|c|c|c|l|c|}
\hline $\begin{array}{c}\text { Step } \\
k\end{array}$ & $\begin{array}{c}\text { Sample } \\
v(t)\end{array}$ & $\delta_{j(k-1)}$ & \multicolumn{1}{|c|}{$w_{j k}$} & \multicolumn{1}{|c|}{$\delta_{j}$} \\
\hline 0 & $\mathrm{v}_{\mathrm{j}}$ & $\begin{array}{c}0 \\
(\mathrm{set})\end{array}$ & 0 & 1 (set) \\
\hline 1 & $\mathrm{v}_{\mathrm{j}}$ & 1 & $\mathrm{w}_{\mathrm{j} 1}=\mathrm{v}_{\mathrm{j}}-\delta_{0}$ & $\delta_{\mathrm{j} 1}=\mathrm{q}\left(\mathrm{w}_{\mathrm{j} 1}\right)$ \\
\hline 2 & $\mathrm{v}_{\mathrm{j}}$ & $\delta_{\mathrm{j} 1}$ & $\begin{array}{l}\mathrm{w}_{\mathrm{j} 2}=\mathrm{w}_{\mathrm{j} 1}+\left(\mathrm{v}_{\mathrm{j}}-\delta_{1}\right) \\
=\mathrm{v}_{\mathrm{j}}-\delta_{0}+\mathrm{v}_{\mathrm{j}}-\delta_{1} \\
=2 \mathrm{v}_{\mathrm{j}}-\delta_{0}-\delta_{1} \\
=2 \mathrm{v}_{\mathrm{j}}-\mathrm{S}_{\mathrm{j} 2}\end{array}$ & $\delta_{\mathrm{j} 2}=\mathrm{q}\left(\mathrm{w}_{\mathrm{j} 2}\right)$ \\
\hline 3 & $\mathrm{v}_{\mathrm{j}}$ & $\delta_{\mathrm{j} 2}$ & $\mathrm{w}_{\mathrm{i} 3}=3 \mathrm{v}_{\mathrm{j}}-\mathrm{S}_{\mathrm{j} 3}$ & $\delta_{\mathrm{j} 3}=\mathrm{q}\left(\mathrm{w}_{\mathrm{j} 3}\right)$ \\
\hline$\vdots$ & $\vdots$ & $\vdots$ & $\vdots$ & $\vdots$ \\
\hline $\mathrm{k}$ & $\mathrm{v}_{\mathrm{j}}$ & $\delta_{\mathrm{i}(\mathrm{k}-1)}$ & $\mathrm{w}_{\mathrm{jk}}=\mathrm{kv}_{\mathrm{j}}-\mathrm{S}_{\mathrm{jk}}$ & $\delta_{\mathrm{jk}}=\mathrm{q}\left(\mathrm{w}_{\mathrm{jk}}\right)$ \\
\hline
\end{tabular}

CEEA Conf. 2014; Paper 107

Canmore, AB; June 8-11, 2014

\section{DisCUSSION}

\subsection{Noise Averaging}

The entire description assumed that the analog signal $\mathrm{v}(\mathrm{t})$ was sampled by a $\mathrm{S} \& \mathrm{H}$ device, and that the sample $v_{j}$ was constant throughout the pulse generation in each sample period $T_{S}$. In practice, the value of the analog discrete sample $v_{j}$ droops (like a leaky memory), and is also subjected to external noise. Another source of the noise is due to clock jitter. The strength of the $\Delta \Sigma$ ADC is that the many pulses tend to average out the noise through the integration process. Such systems have a comb filter transfer characteristic [Kins13a].

\subsection{How Many Pulses per $T_{\mathrm{s}}$ ?}

The number of pulses per sample period $T_{S}$, should be as large as possible in order to achieve accurate convergence to each sample $v_{j}$, and to smooth out the variability in $v_{j}$ between the samples. The oversampling is often $M=64$ or higher (sometimes 32,768 ). Since $f_{S}=2 f_{B}$, and $f_{O S}=M f_{S}$, then $f_{O S}=64 \times 2 f_{B}$.

\subsection{Are All the Pulses Needed?}

The large number of bipolar pulses are needed to move the quantization noise out of the band of interest. To extract the signal from the pulse stream, we can use a LPF to reduce the bandwidth back to the original value of $B$. While the pulse stream is shifted into the filter at the oversampling frequency $f_{O S}$, the computations have to performed at the sampling rate, $f_{S}$.

For example, since the telephone-quality speech has a sampling rate of $f_{S}=8 \mathrm{ksps}$, the oversampling is $f_{S},=M f_{S}=$ $64 \times 8=512 \mathrm{ksps}$.

\subsection{Pulse Storage}

Direct storage of the $+1 \mathrm{~s}$ and $-1 \mathrm{~s}$ is not necessary, as we can convert the bipolar stream into a unipolar $1 \mathrm{~s}$ and $0 \mathrm{~s}$. If necessary, the original stream can be reconstructed without any loss of information.

\subsection{Actual Devices}

Many manufacturers produce $\Delta \Sigma$ ADCs. For example, Analog Devices AD7760 ADC provides 24 bit resolution at up to 2.5 Msps. It uses three FIR LPFs, and has integral nonlinearity of $0.00076 \%$ (at $\$ 25$ each)!

Microchip Technology has a 16-bit MCP3425 lowpower (145 $\mu \mathrm{A} @ 15 \mathrm{sps}) \mathrm{ADC}$ at a cost of $\$ 2$.

The devices have many parameters [Kins13, [21\}, including noise measures such as signal-to-noise ratio (SNR), spurious-free dynamic range (SFDR), and signal-to- 
noise-and-distortion (SINAD) ratio. If the $\mathrm{AC} \mathrm{SNR}=79$ $\mathrm{dBFS}$, the rms noise is below the FS, or the power ratio is $10^{79 / 10}$, or the voltage ratio is $10^{79 / 20}$. Notice that this is not the same as the DC dynamic range calculation from Eq. (7). For example, for $N=16$, the calculated value is $\mathrm{SNR}_{\mathrm{DC}}=$ $98 \mathrm{~dB}$.

SINAD is often worse than SNR because it includes the harmonics of the input signal. SINAD is also useful to establish the effective number of bits (ENOB)

$$
E N O B=\frac{S I N A D-1.76}{6.02}
$$

\section{Concluding ReMARKS}

This paper provides a new approach to teaching the $\Delta \Sigma$ signal conversion. The key ideas behind the standard serial analog-to-digital converters (ADCs) are classified as pulsewidth-modulation (PWM), and frequency-modulation (FM) ADCs. The discussion of various ADC issues includes oversampling and its ability to reduce the unavoidable quantization noise in ADC. This prepares the ground for another strategy to reduce quantization noise through a non-linear transformation that results from a negative feedback (the $\Delta \Sigma$ ADC).

The oversampling leads to linear quantization noise reduction. Oversampling with feedback (either the firstorder or higher-order) leads to a non-linear noise reduction by skewing most of the noise energy away from the baseband region to higher frequencies. This strategy is a special case of a larger dithering strategy to spread the noise over a much larger bandwidth, either linearly or nonlinearly (e.g., [8], [31], [36]).

Another advancement in the $\Delta \Sigma$ converters is the shift from integer oversampling $M$ to fractional oversampling. As with the fractional calculus in control and signal processing, the fractional $\Delta \Sigma$ converters can expand their flexibilities considerably (e.g., [28]).

Based on the above setting, the main reason for this paper is the discovery of a simple, well-understandable principle behind the formation of the next bipolar pulse in the pulse stream. The principle is easy to verify on paper, easy to implement on a simple computer, and easy to implement on a microcontroller for embedded systems.

\section{Acknowledgements}

Special thanks go to the Department of Electrical and Computer Engineering at the University of Manitoba for partial financial support of this project.

\section{References}

[1] Pervez M. Aziz, Henrik V. Sorensen, and Jan Van der Spiegel, "An overview of sigma-delta converters: How a 1-bit ADC achieves more than 16-bit resolution," IEEE Signal Processing Mag., vol. 1, no. 1, pp. 61-84, Sept 1996.

[2] Olli Aumala, Dithering in Analogue-to-Digital Conversion. Doctoral Dissertation. Tempere, Finland: Tampere University of Technology. Publication 325, May 7, 2001, 48 pp.

[3] Arnold S. Berger, Embedded Systems Design: An Introduction to Processes, Tools, and Techniques. Lawrence, KS: CMP Books, 2002, 237 pp. \{ISBN: 978-1-57820073-3

[4] Fredrick M. Cady, Software and Hardware Engineering: Assembly and C Programming for the Freescale HCS12 Microcontroller. Oxford, UK: Oxford University, 2008 (2nd ed.), 750 pp. Includes a CDROM with CodeWarrior Studio for HCS12, v. 4.5.

[5] James C. Candy and Gabor C. Temes, "Oversampling methods for A/D and D/A conversion," in James C. Candy and Gabor C. Temes (eds.), Oversampling Delta-Sigma Data Converters: Theory, Design, and Simulation. New York, NY: Wiley/IEEE, pp. 1-25, 1991, 512 pp. \{ISBN: 978-0-87942-285-1\}

[6] Cassius C. Cutler, "Differential quantization of communication signals," US Patent 2,605,361, July 29, 1952.

[7] Edmond M. Deloraine, S. Van Mierlo, and Boris Derjavitch, "Methode de syséme de transmission par impulsions," French Patent 932,140, August 1946. Also "Communication system utilizing constant amplitude pulses of opposite polarities," British Patent 627,262, an US Patent 2,629,857A, Feb 24, 1953. First invented in the Paris lab of the International Telephone and Telegraph Corporation.

[8] Roberto Etchenique and J. Aliaga, "Resolution enhancement by dithering," Am. J. Phys., vol. 72, no. 2, pp. 159-163, Feb 2004.

[9] Günther Gridling and Bettina Weiss, Introduction to Microcontrollers. Vienna, AT: Vienna University of Technology, February 2, 2007 (Version 1.4), 175 pp. Available as of September 2013 from http://wwwold.ecs.tuwien.ac.at/lehre/Microcontroller/ Docs/Lecture/Microcontroller.pdf

[10] Max W. Hauser, "Principles of oversampling A/D conversion," J. Audio Eng. Soc., vol. 39, no.1/2, pp. 326, Jan/Feb 1991.

[11] F. de Jager, "Delta modulation: A method of PCMN transmission using the one-unit code," Pillips Research Reports, vol. 7, pp. 542-546, 1952.

[12] Walt Kester, The Data Conversion Handbook. Analog Devices, 2004, 1140 pp. \{ISBN 0-916550-27-3\}

[13] Walt Kester, ADC Architectures III: Sigma-Delta ADC Basics. MT-22 Tutorial. Norwood, MA: Analog 
Devices, Aug 2008, 12 pp. Available as of Dec 31, 2013 from

http://www.analog.com/static/importedfiles/tutorials/MT-022.pdf

[14] Walt Kester, ADC Architectures IV: Sigma-Delta ADC Advanced Concepts and Applications. MT-23 Tutorial. Norwood, MA: Analog Devices, Aug 2008, 11 pp. Available as of Dec 31, 2013 from http://www.analog.com/static/importedfiles/tutorials/MT-023.pdf

[15] Walt Kester, Taking the Mystery out of the Infamous Formula, $S N R=6.02 \mathrm{~N}+1.76 \mathrm{~dB}$, and Why You Should Care. MT-001 Tutorial. Norwood, MA: Analog Devices, Oct 2008, 7 pp. Available as of Dec 31, 2013 from http://www.analog.com/static/importedfiles/tutorials/MT-001.pdf

[16] Witold Kinsner, Microcontroller, Microprocessor, and Microcomputer Interfacing for Real-Time Systems. Lecture Notes. Winnipeg. MB: University of Manitoba, 2013, 643 pp.

[17] Witold Kinsner, Laboratories for Microcontroller, Microprocessor, and Microcomputer Interfacing for Real-Time Systems. Lab Notes; 2013, 102 pp.

[18] Witold Kinsner, The HCS12 Microcontroller: A Tutorial. Winnipeg. MB: University of Manitoba, 2013, $66 \mathrm{pp}$.

[19] Haideh Khorramabadi, EE247 Analog-Digital Interfaces in VLSI Technology: Lecture Slides. Univ. Berkeley, CA: California at Berkeley, Department of Electrical Engineering and Computer Science, 2010. (Previous version 2007.) Available as of Dec 31, 2013 from http://inst.eecs.berkeley.edu/ ee247/fa10/lectures.html http://inst.eecs.berkeley.edu/ ee247/fa07/lectures.html

[20] Robert Lacoste, "Playing with high-speed ADCs," Circuit Cellar, no. 259, pp. 52-59, Feb 2012.

[21] Robert Lacoste, "Sigma-delta modulators 101," Circuit Cellar, no. 261, pp. 42-50, Apr 2012.

[22] Robert Lacoste, "No fear with FIR: Put a FIR filter to work," Circuit Cellar, no. 207, pp. 70-78, Oct 2007.

[23] David Ludington, "High-accuracy voltage reference using PWM (Part 1): Pulse -width modulation theory," Circuit Cellar, no. 255, pp. 36-38, Oct 2011.

[24] David Ludington, "High-accuracy voltage reference using PWM (Part 2): Hardware design," Circuit Cellar, no. 256, pp. 18-20, Nov 2011.

[25] Richard G. Lyons, Understanding Digital Signal Processing. Upper Saddle River, NJ: Prentice Hall, 2005 (2nd ed.) (2001, 1st ed.) 517 pp. \{ISBN: 0-20163467-8, hbk\}
[26] Muhammad Ali Mazidi and Danny Causey, HCS12 Microcontroller and Embedded Systems using Assembly and C with Code Warrior. Upper Saddle River, NJ: Pearson/ Prentice Hall, 2009, 738 pp. \{ISBN-13: 978-0-13-607229-4; \$135.83\}

[27] Mike Perkins, Delta-sigma converters: Modulation (Part 1) and Filtering, decimation and simulation (Part 2). CardinalPeak, 2010. Available as of December 30, 2013 from http://www.cardinalpeak.com/blog/delta-sigmaconverters-modulation/ http://www.cardinalpeak.com/blog/delta-sigmaconverters-filtering-decimation-and-simulations/

[28] Tom A. D. Riley, Miles A. Copeland, and Tad A. Kwasniewski, "Delta-sigma modulation in fractional- $N$ frequency synthesis," IEEE J. Solid-State Circuits, vol. 28, no. 5, pp. 553-559, May 1993.

[29] José Barreiro da Silva, High-Performance DeltaSigma Analog-to-Digital Converters. PhD Thesis. Corvallis, OR: Oregon State University, July 14, 2004, $152 \mathrm{pp}$.

[30] Steven W. Smith, Digital Signal Processing: A Practical Guide for Engineers and Scientists. Burlington, MA: Newness, 2003, 650 pp. \{ISBN: 0750674-44-X; pbk + CD-ROM with the eBook version\}

[31] David Tweed, "Digital processing in an analog world (Part 3): Dithering your conversion," Circuit Cellar, no. 101, pp. 68-73, Dec 1998.

[32] Jonathan W. Valvano, Embedded Microcomputer Systems: Real Time Interfacing. Stamford, CT: CENGAGE Learning, 2012 (3rd ed.), 793 pp. \& CDROM. This book covers design methodologies with examples, using the Motorola 9S12 machine, with many practical examples. The CD-ROM includes a simulator to show internal and I/O activities, and many links to data sheets. \{ISBN 978-1-111-42625-5\}

[33] Han-Way Wang, HCS12/9S12: An Introduction to Software and Hardware Interfacing. New York, NY: Delmar Cengage Learning, 2009 ( $2^{\text {nd }}$ ed.), 880 pp.

[34] Bernard Widrow and Istvan Kollar, Quantization Noise: Roundoff Error in Digital Computation, Signal Processing, Control, and Communications. Cambridge, UK: Cambridge University, 2008, 778 pp. \{ISBN: 978-0521886710; hbk\}

[35] Bruce A. Wooley, "The evolution of oversampling analog-to-digital converters," in Solid-State Circuits Soc. Conf. (Santa Clara, CA; March 22, 2012), Presentation, 2012, 65 pp.

[36] S. Zozor and P.-O. Amblard, "Noise-aided processing: Revisiting dithering in a $\Sigma \Delta$ quantizer," IEEE Trans. Sign. Proc., vol. 53, no. 8, pp. 3202-3210, Aug 2005. 